\title{
Política de Investigação Científica para a Saúde em Portugal: II - Factos e Sugestões
}

\section{Scientific Research Policy for Health in Portugal: II - Facts and Suggestions}

\author{
Cátia Sá GUERREIRO ${ }^{1}$, Zulmira HARTZ¹, Luís SAMBO ${ }^{1,2}$, Claúdia CONCEIÇÃO ${ }^{1}$, Gilles DUSSAULT¹, \\ Giuliano RUSSO ${ }^{1}$, Miguel VIVEIROS ${ }^{1}$, Henrique SILVEIRA ${ }^{1}$, Pedro PITA BARROS ${ }^{3}$, Paulo FERRINHO ${ }^{1}$ \\ Acta Med Port 2017 Mar;30(3):233-242 - https://doi.org/10.20344/amp.8012
}

RESUMO

Tendo Portugal superado o atraso científico estrutural vivido até há cerca de três décadas, a análise desta matéria à luz das políticas de investigação definidas a nível global e europeu mostra que há ainda um longo caminho a percorrer quando se fala em investimento global em Investigação \& Desenvolvimento. A investigação para a saúde em Portugal tem tido tutela partilhada entre a Fundação para a Ciência e Tecnologia e o Instituto Nacional de Saúde Doutor Ricardo Jorge, sendo que esta matéria não tem sido uma prioridade - a realidade demonstra a não existência de um plano de investigação científica para a saúde em Portugal, o qual possa pôr em franca articulação os diferentes atores intervenientes. As orientações estratégias do Plano Nacional de Saúde 2004 - 2010 são as que mais se aproximam de uma política de investigação para a saúde para Portugal sem que, no entanto, as questões então abordadas tenham sido desenvolvidas de uma forma abrangente ou, à posteriori, implementadas pelas instituições que têm responsabilidades sobre a investigação científica no setor da saúde. Na convicção de que adoptar uma estratégia de incentivo à investigação para a saúde consiste uma mais-valia para o sistema de saúde português, os autores propõem neste trabalho cinco sugestões estratégicas em matérias de investigação para a saúde em Portugal.

Palavras-chave: Investigação Biomédica; Investigação para a Saúde; Investigação sobre Serviços de Saúde; Portugal; Programas Nacionais de Saúde; Saúde Pública

\section{ABSTRACT}

After more than 40 years of democracy and 30 years of European integration, Portugal has bridged the research gap it had previously. However, when compared to global and European research policies, Portugal still has a long way go regarding investment in research and development. Health Research in Portugal has been managed by the Fundação para a Ciência e Tecnologia and the National Health Institute Doctor Ricardo Jorge, and it has not been a political priority, emphasized by the absence of a national scientific research plan for health, resulting in a weak coordination of actors in the field. The strategic guidelines of the $2004-2010$ National Health Plan are what comes closest to a health research policy, but these were not implemented by the institutions responsible for scientific research for the health sector. Trusting that adopting a strategy of incentives to stimulate health research is an added-value for the Portuguese health system, the authors present five strategic proposals for research in health in Portugal.

Keywords: Biomedical Research; Health Services Research; National Health Programs; Portugal; Public Health

\section{INTRODUÇÃO}

O presente trabalho pretende apresentar uma visão global da política de investigação científica para a saúde em Portugal. Partindo do enquadramento da experiência portuguesa em matéria de política de investigação científica efetuado na primeira parte do presente artigo, ${ }^{1}$ aprofunda-se agora especificamente a investigação científica para a saúde, apresentando alguns dos atores envolvidos. Da reflexão efetuada discorre um conjunto de sugestões que pretende contribuir para a definição de uma política de investigação para a saúde em Portugal.

\section{A INVESTIGAÇÃO PARA A SAÚDE EM PORTUGAL, ATORES E AGENDA}

Tendo Portugal superado o atraso científico estrutural de que foi alvo até há cerca de três décadas, ${ }^{2}$ a análise deste progresso à luz das políticas de investigação definidas a nível global e europeu mostra que há ainda um longo caminho a percorrer quando se fala em investimento global em Investigação \& Desenvolvimento, sendo que o país tem evidenciado uma capacidade insuficiente para conceber e implementar, de forma participativa, uma política de investigação e ciência. ${ }^{3,4}$

Até à década de 90 , a realidade da ciência e tecnologia em Portugal era caracterizada por uma dimensão e articulação consideravelmente reduzidas. Apenas a partir de 1996 as instituições científicas começam a ser sujeitas a avaliações independentes, o que constituiu um acontecimento chave no âmbito da efetiva abertura da comunidade científica e da construção do sistema científico português. ${ }^{1,2}$ Heitor (2015) identifica seis principais períodos que caracterizaram a evolução recente do sistema português de Ciência e Tecnologia² (Fig. 1).

\section{A investigação para a saúde em Portugal}

A investigação para a saúde em Portugal seguiu naturalmente a tendência da investigação científica nacional

\footnotetext{
1. R\&D Unit. Instituto de Higiene e Medicina Tropical. Universidade Nova de Lisboa. Lisboa. Portugal.

2. Ministério da Saúde. Governo da República Popular de Angola. Luanda. Angola.

3. Nova School of Business and Economics. Universidade Nova de Lisboa. Lisboa. Portugal.

$\square$ Autor correspondente: Cátia Sá Guerreiro. cguerreiro@ihmt.unl.pt

Recebido: 05 de julho de 2016 - Aceite: 07 de fevereiro de 2017 | Copyright $\odot$ Ordem dos Médicos 2017
} 


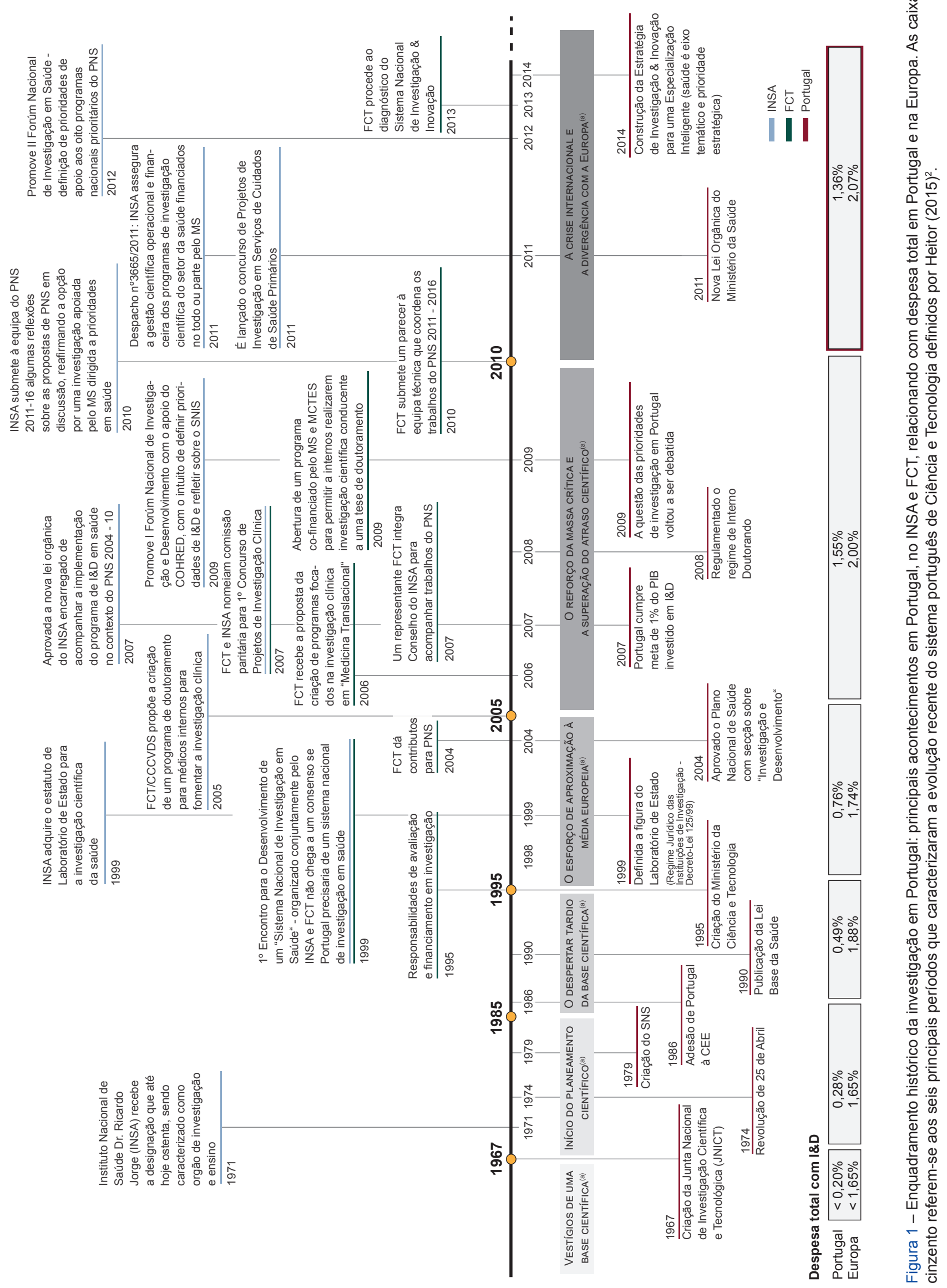


descrita na primeira parte do presente artigo. ${ }^{1}$ Os investigadores em Portugal na área de Ciências Médicas e da Saúde aumentaram em número absoluto de 4223 em 2000 para 13493 em 2012 (OCDE, 2014), ${ }^{2}$ sendo que a maior amplitude de variação ocorreu até 2010. Por outro lado, relativamente a bolsas de doutoramento atribuídas pela Fundação para a Ciência e a Tecnologia (FCT) na área das Ciências Médicas e da Saúde, verifica-se que estas eram 46 em 1998, aumentando para 80 em 2000 e para 243 em 2007, sofrendo depois um decréscimo nos anos seguintes, registando-se em 2012 um total de 200 bolsas atribuídas à referida área de investigação científica. ${ }^{2}$

Segundo o Plano Nacional de Saúde (PNS) 2004 $2010,{ }^{5}$ o sistema de saúde português não tem sido tradicionalmente suportado por uma forte componente de conhecimento científico baseado no contexto e produção nacional.

A mesma fonte refere que os financiamentos estatais, e portanto, a coordenação da investigação para a saúde não estão maioritariamente sob o controlo do Ministério da Saúde mas sim do Ministério da Ciência e do Ensino Superior, através de instituições como a FCT, salientando o papel fulcral das instituições da sociedade civil na promoção de uma investigação virada para os problemas de saúde no país. Instituições internacionais, nomeadamente as da União Europeia (UE), são referidas como promotoras de projetos de investigação em redes internacionais, abordando as grandes problemáticas da saúde na UE, com importância em termos temáticos, em termos de cultura de colaboração interinstitucionais e em termos de desenvolvimentos metodológicos.

No início do novo milénio, foram publicados dados de 2001 pelo Observatório da Ciência e Ensino Superior, os quais revelam que as ciências da saúde representaram apenas $10,5 \%$ da despesa nacional em I\&D, ficando em último lugar entre as diferentes áreas científicas (relativamente, por exemplo, aos $11,1 \%$ das ciências agrárias e veterinárias, aos $15,3 \%$ das ciências naturais ou aos $25,3 \%$ das ciências da engenharia e tecnologias). ${ }^{5,6}$ Em 2013, as ciências médicas e da saúde representaram $12 \%$ da despesa em I\&D, em exaequo com as ciências sociais. Abaixo destas apenas as Humanidades, domínio científico e tecnológico que representou $7 \%$ da despesa. Ciências da engenharia e tecnologias e Ciências exatas e naturais representaram nesse ano respetivamente $42 \%$ e $24 \%$ da despesa nacional em I\&D. ${ }^{7}$ No cenário europeu, a despesa em I\&D dos sectores de execução do Estado, Ensino Superior e Instituições Privadas Sem Fins Lucrativos (IPSFL) em percentagem do PIB, por área científica, no que concerne especificamente à área das ciências médicas e da saúde, coloca Portugal na cauda da Europa com 0,11\%, apenas acima da Irlanda e do Luxemburgo. A Dinamarca, com uma despesa de $0,36 \%$ nesta área científica, regista o valor mais elevado. ${ }^{8}$ No mesmo ano, considerando a despesa em I\&D em percentagem do PIB, dos sectores de execução do Estado, Ensino Superior e IPSFL, Portugal apresenta um valor de $0.69 \%$, sendo que apenas a Finlândia, a Suécia e a Dinamarca registam valores de despesa acima de $1 \%{ }^{8}$
Por altura da redação do PNS 2004 - 2010 a investigação não era contemplada como uma necessidade para o sistema de saúde nacional, nem sequer de prioridade secundária, tendo as atividades de investigação um baixo peso nos critérios contemplados na evolução dos profissionais de saúde nas suas carreiras. ${ }^{5,6}$ Por outro lado, e segundo a mesma fonte, verificava-se então ainda uma grande diversidade temática sem uma priorização adequada, uma grande dispersão de recursos e um persistente subfinanciamento.

O debate sobre políticas de investigação para a saúde em Portugal tem sido inconclusivo e sobretudo inconsequente, sendo que esta matéria carece de uma análise aprofundada que permita ler os dados disponíveis numa perspetiva de melhoria futura (Nos trabalhos de extensão do atual PNS até 2020, está em curso a realização de uma nova análise especializada sobre investigação para a saúde: http://pns.dgs.pt/roteiros-de-intervencao-do-plano-nacional-de-saude/).

Embora na lei n. ${ }^{\circ}$ 56/79, que criou o Serviço Nacional de Saúde (SNS), seja referido como órgão central de natureza instrumental o Departamento de Ensino e Investigação, tendo como missão "promover e coordenar as atividades de ensino e investigação no campo da saúde" e "propor as medidas destinadas à uniformização de objetos de idênticas atividades dependentes de outros ministérios", a investigação para a saúde em Portugal tem tido tutela partilhada entre a FCT e o Instituto Nacional de Saúde Doutor. Ricardo Jorge (INSA).

\section{A FCT e a investigação em saúde}

A FCT é o organismo que, tutelado pelos ministérios que têm assumido a Ciência e Tecnologia (geralmente em associação com o Ensino Superior), tem gerido a política científica em Portugal, apoiada por quatro conselhos científicos (CC) com uma função consultiva, nomeadamente:

- Ciências Exatas e da Engenharia;

- Ciências da Vida e da Saúde (CCCVDS);

- Ciências Naturais e do Ambiente;

- Ciências Sociais e Humanidades. ${ }^{4}$

A FCT iniciou atividades em agosto de 1997 sucedendo à Junta Nacional de Investigação Científica e Tecnológica (JNICT). Desde 1 de março de 2012, a FCT sucede à UMIC - Agência para a Sociedade do Conhecimento, I.P. na responsabilidade pela coordenação das políticas públicas para a Sociedade da Informação em Portugal. Em 1 de outubro de 2013, assumiu também as atribuições e competências da Fundação para a Computação Científica Nacional (FCCN).

A missão da FCT é "Promover continuadamente o avanço do conhecimento científico e tecnológico em Portugal, atingir os mais elevados padrões internacionais de qualidade e competitividade em todos os domínios científicos e tecnológicos, e estimular a sua difusão e contribuição para a sociedade e o tecido produtivo". De acordo com o site institucional (acedido a 16.06.2016), a FCT prossegue a sua missão: 
a) Através da atribuição, em concursos com avaliação por pares, de bolsas e contratos a investigadores, financiamento a projetos de investigação e desenvolvimento, apoio a centros de investigação competitivos e a infraestruturas de investigação de ponta.

b) Assegurando a participação de Portugal em organizações científicas internacionais, promove a participação da comunidade científica nacional em projetos internacionais e estimula a transferência de conhecimento entre centros de investigação e a indústria.

c) Coordenando as políticas públicas para a Sociedade da Informação e do Conhecimento em Portugal, em estreita colaboração com organizações internacionais.

d) Assegurando o desenvolvimento dos meios nacionais de computação científica, promovendo a instalação e utilização de meios e serviços avançados e a sua articulação em rede.

De uma posição em que a ciência serviria para aumentar o conhecimento, motivada pela curiosidade científica, a FCT tem evoluído para uma posição mais próxima da política científica do Reino Unido, e mais recentemente da UE, de que a ciência deveria servir para aumentar a relevância nacional e a competitividade em termos internacionais e de que os financiamentos públicos não deveriam apoiar investigação que não seja economicamente útil (i.e. com impacto social) ou de referência - criação de valor através da exploração económica do conhecimento. ${ }^{8}$

Ao invés do anterior encorajamento de muitos pequenos projetos executados por pequenos grupos espalhados por muitas unidades de investigação, com a finalidade de aumentar o número de cientistas de base académica, a FCT tem progredido para uma política de financiamento de um menor número de projetos e equipas de investigação de maiores dimensões, integrando várias vertentes de investigação em objetivos de referência comum, facilitando a mobilidade entre o tecido educativo e empresarial. Esta abordagem levou também à redução do número de bolsas de estudo para programas de doutoramento tradicionais, para as concentrar em novos programas de doutoramento que assegurem a aquisição de competências comportamentais apreciadas pelo tecido empresarial, mais apropriadas para que o doutorado crie a sua oportunidade de trabaIho ou se integre mais facilmente no mercado de trabalho internacional. $^{9}$

Considerando que o grosso do financiamento tem sido dirigido para a atividade científica do projeto, é de salientar que têm aparecido indícios de uma maior preocupação com o financiamento de infraestruturas e equipamentos, bem como com a contratação de pessoal técnico (p.e. técnicos de laboratório) e administrativo. ${ }^{9}$

A FCT financia projetos de investigação 'em' e 'para' a saúde e tem aberto concursos para financiamento de projetos de investigação de dois em dois anos desde 2000 e todos os anos desde 2008. Recentemente definiu o Portuguese Roadmap of Research Infrastructures 2014-2020 que apoia o estabelecimento de nove infraestruturas para a investigação em ciências biológicas e médicas. ${ }^{10}$
A participação da FCT nas atividades na área da investigação para a saúde já vem de longa data com a criação do CCCVDS. Inicialmente esta participação incidiu no financiamento de projetos, de infraestruturas e de bolseiros a vários níveis, para além da criação dos laboratórios associados, e das unidades de investigação acreditadas pela FCT. ${ }^{11}$

Alguns elementos da história deste envolvimento são descritos na Fig. 1.

\section{O INSA e a investigação em saúde}

INSA é uma instituição fundada em 1899. No ano de 1971, no âmbito de uma reforma global dos Serviços de Saúde, o INSA recebeu a designação que atualmente ostenta, e foi caracterizado como órgão de investigação e ensino (Dec-Lei 413/71 de 27 de setembro). Neste mesmo Decreto-Lei, artigo 22, lê-se que compete ao INSA coordenar, no âmbito do Ministério da Saúde (MS), as atividades de investigação no setor da saúde; efetuar, promover e estimular a realização de estudos e trabalhos de investigação científica relativa à saúde em colaboração com os demais serviços do ministério. Estas responsabilidades são reconfirmadas em novas leis orgânicas em 1993, 2007 e 2011. O Decreto-Lei n. ${ }^{0} 125 / 99$ de 20 de Abril veio reforçar as responsabilidades na área da investigação ao atribuir-lhe o estatuto de Laboratório de Estado (um Laboratório de Estado é uma instituição pública de investigação criada e mantida com o propósito explícito de prosseguir objetivos da política científica e tecnológica adotada pelo Governo, mediante a prossecução de investigação científica e desenvolvimentos tecnológico e de outras atividades científicas e técnicas que as respetivas leis orgânicas prevejam. São formalmente consultados pelo Governo sobre a definição dos programas e instrumentos da política científica e tecnológica nacional. $)^{12}$ do setor da saúde..$^{13}$ Enquanto organismo do MS, a sua missão e atribuições estão descritas na Tabela 1.

A capacidade de intervenção do INSA na indução da investigação para a saúde por instituições do Serviço Nacional de Saúde tem sido limitada por financiamentos escassos face ao necessário, pelo que grande número de projetos de investigação são autofinanciados pelas entidades públicas ou financiados por fontes externas ao MS como a FCT, o Quadro de Referência Estratégica Nacional (QREN), fundações, fundos europeus, indústria, entre outros. ${ }^{13}$

A FCT e o INSA têm vindo a discutir a questão da definição de áreas prioritárias na investigação para a saúde. A FCT tem defendido uma perspetiva bottom-up, i.e., que consiste em considerar que a investigação de valor nasce da iniciativa individual e da curiosidade dos bons investigadores, muitas vezes oriundos de quadrantes inesperados, e não sendo orientada a partir de diretrizes superiores. Esta contrasta com a perspetiva top-down do INSA, em que é necessário definir áreas prioritárias de acordo com as políticas de Saúde Pública. A FCT tem considerado que a perspetiva top-down não deve dominar o conjunto de atividades de investigação, devendo restringir-se a aspetos de carácter 
Tabela 1 - Missão e atribuições do Instituto Nacional de Saúde Doutor Ricardo Jorge de acordo com a Lei Orgânica do Ministério da Saúde, DL n. ${ }^{\circ} 124 / 2011$, de 29 de Dezembro

\begin{tabular}{|c|c|}
\hline Missão & Atribuições \\
\hline $\begin{array}{l}\text { É o laboratório do Estado que } \\
\text { tem por missão contribuir para } \\
\text { ganhos em saúde pública } \\
\text { através da investigação e } \\
\text { desenvolvimento tecnológico, } \\
\text { atividade laboratorial de } \\
\text { referência, observação } \\
\text { da saúde e vigilância } \\
\text { epidemiológica, bem como } \\
\text { coordenar a avaliação externa } \\
\text { da qualidade laboratorial, } \\
\text { difundir a cultura científica, } \\
\text { fomentar a capacitação e } \\
\text { formação e ainda assegurar } \\
\text { a prestação de serviços } \\
\text { diferenciados, nos referidos } \\
\text { domínios. }\end{array}$ & $\begin{array}{l}\text { a) Promover e desenvolver a atividade de investigação científica orientada para as necessidades } \\
\text { em saúde pública, procedendo à gestão científica, operacional e financeira dos programas de } \\
\text { investigação do sector da saúde pública, e capacitar investigadores e técnicos; } \\
\text { b) Promover, organizar e coordenar programas de avaliação, no âmbito das suas atribuições, } \\
\text { nomeadamente na avaliação externa da qualidade laboratorial e colaborar na avaliação da } \\
\text { instalação e funcionamento dos laboratórios privados que exerçam atividade no sector da saúde; } \\
\text { c) Assegurar o apoio técnico-normativo aos laboratórios de saúde pública; } \\
\text { d) Prestar assistência diferenciada em genética médica para prevenção e diagnóstico, em } \\
\text { serviços laboratoriais, bem como planear e executar o programa nacional de diagnóstico } \\
\text { precoce; } \\
\text { e) Colaborar na realização de atividades de vigilância epidemiológica de doenças, transmissíveis } \\
\text { e não transmissíveis, e desenvolver ou validar instrumentos de observação em saúde, no âmbito } \\
\text { de sistemas de informação, designadamente garantindo a produção e divulgação de estatísticas } \\
\text { de saúde pública, e promovendo os estudos técnicos necessários, sem prejuizo das atribuições } \\
\text { da DGS e da ACSS, I. P., nesta matéria; } \\
\text { f) Proceder à monitorização do consumo de aditivos e da exposição da população a } \\
\text { contaminantes e outras substâncias potencialmente nocivas presentes nos alimentos, incluindo } \\
\text { os ingredientes alimentares cujo nível de ingestão possa colocar em risco a saúde dos } \\
\text { consumidores; } \\
\text { g) Assegurar a recolha, compilação e transmissão para a Autoridade Europeia de Segurança } \\
\text { Alimentar dos dados analíticos relativos à composição, incluindo contaminantes e outras } \\
\text { substâncias químicas, dos géneros alimentícios e alimentos para animais; } \\
\text { h) Avaliar a execução e resultados das políticas, do PNS e programas de saúde do MS; } \\
\text { i) Prestar assessoria científica e técnica a entidades públicas e privadas, nas suas áreas de } \\
\text { atuação; } \\
\text { j) Desenvolver açães de cooperação nacional e internacional, de natureza bilateral ou } \\
\text { multilateral, no âmbito das atribuicões que prossegue. }\end{array}$ \\
\hline
\end{tabular}

geral, tal como a criação de infraestruturas científicas e de programas de formação específicos em áreas científicas consideradas prioritárias. ${ }^{10}$ As duas perspectivas porém não se excluem mutuamente, podendo completar-se, pois, a abordagem top-down poderia enquadrar as iniciativas bottom-up.

\section{Agenda nacional de investigação para a saúde}

Apesar dos propósitos e tentativas de construção de uma agenda nacional de investigação em saúde, sobretudo associada à diretriz do PNS, segundo a qual os programas de saúde específicos deverão explicitar as principais lacunas de conhecimento a requerer mais esforço de I\&D, ${ }^{14}$ a realidade é que nunca foi finalizada uma agenda nacional de investigação e o INSA não conseguiu dinamizar as propostas e orientações sobre investigação aprovadas no âmbito dos Planos Nacionais de Saúde.

O Diagnóstico do Sistema Nacional de Investigação e Inovação (SNI\&I) português, ${ }^{15}$ efetuado pela FCT em 2013 e referido na primeira parte do presente artigo $^{1}$, refere a importância do setor da saúde no sistema científico e tecnológico nacional, tendo-se verificado um crescimento médio anual da distribuição da despesa de I\&D de 45,3\% na área das Ciências Médicas e da Saúde. ${ }^{16}$

No âmbito da Estratégia de Investigação e Inovação para uma Especialização Inteligente (EI\&I), definida em Novembro de 2014, na sequência do Diagnóstico do SNI\&I, o desafio que se coloca hoje a Portugal no sector da saúde é o da definição de prioridades estratégicas, de acordo com os recursos disponíveis, as áreas de excelência e os problemas de saúde que carecem de maior enfoque pela sua prevalência e pelos custos que representam para os indivíduos e para o Estado. Refere ainda que esta etapa é essencial para permitir, por um lado, um maior bem-estar das populações e melhoramento da saúde pública, e por outro, uma resposta sustentável às oportunidades geradas pelos mercados, tornando o país mais competitivo no sector da saúde. ${ }^{16}$

\section{Outros atores relevantes}

As agendas de investigação para a saúde têm sido marcadas também pelos contributos da Entidade Reguladora da Saúde (ERS), por várias entidades do Ministério da Saúde e de Universidades, Fundações e outras Organizações da Sociedade Civil, incluindo o setor empresarial. Por exemplo, e tendo por base apenas a Lei de Bases da Saúde de 2011, outros organismos do MS para além do INSA, com missões muito distintas, têm nas suas atribuições atividades relacionadas com a investigação para a saúde, como se pode ver na Tabela 2.

É de salientar o papel da Comissão de Ética para a Investigação Clínica (Portaria 57/2005 de 20 de Janeiro) bem como as Comissões de Ética nas diferentes unidades de saúde (DL 97/95 de 10 de Maio). São ainda de realçar algumas medidas avulsas, como a criação da Comissão de Fomento da Investigação em Cuidados de Saúde que funcionou entre 1991 e 2008. Na forma de projetos, apoio a atividades de formação, financiamentos ou prémios estas 
iniciativas não têm, até agora, sido estruturantes do debate sobre políticas de investigação para a saúde, com exceção dos contributos de algumas fundações, da ERS e do Health Cluster Portugal (HCP).

\section{As fundações}

As fundações, através de bolsas, prémios, concursos gerais ou específicos - particularmente as Fundações Calouste Gulbenkian, Luso Americana e Champalimaud - têm tido um contributo particularmente importante para a internacionalização da investigação em saúde em Portugal. ${ }^{17}$

Recentemente, a Fundação Calouste Gulbenkian comissionou um relatório sobre "Um Futuro para a Saúde" em Portugal. ${ }^{18}$ Este relatório destaca que a investigação biomédica teve progressos notáveis em Portugal na última década, devido a uma intervenção intensiva na educação científica, nomeadamente através de doutoramentos internacionais e da criação dos chamados 'Laboratórios Associados' do Ministério da Ciência. No entanto, considera que a articulação entre a investigação básica e a investigação aplicada e a promoção de uma 'medicina de translação' mais eficaz ainda não se concretizaram de forma satisfatória, o que, de certo modo, reflete o declínio generalizado do chamado 'médico-cientista'. Deveria também ser dada maior atenção ao desenvolvimento de programas de investigação no interior das unidades de cuidados de

Tabela 2 - Organismos do Ministério da Saúde com atribuições relacionadas com Investigação \& Desenvolvimento, de acordo com a Lei Orgânica do Ministério da Saúde, DL n. ${ }^{\circ} 124 / 2011$, de 29 de Dezembro

\begin{tabular}{|c|c|}
\hline & Missão \\
\hline $\begin{array}{l}\text { Direção-Geral da } \\
\text { Saúde (DGS) } \\
\text { Art. } 12^{\circ}\end{array}$ & $\begin{array}{l}\text { Regulamentar, orientar e coordenar as atividades de } \\
\text { promoção da saúde e prevenção da doença, definir } \\
\text { as condições técnicas para a adequada prestação } \\
\text { de cuidados de saúde, planear e programar a política } \\
\text { nacional para a qualidade no sistema de saúde, bem } \\
\text { como assegurar a elaboração e execução do Plano } \\
\text { Nacional de Saúde (PNS) e, ainda, a coordenação das } \\
\text { relações internacionais do MS. }\end{array}$ \\
\hline 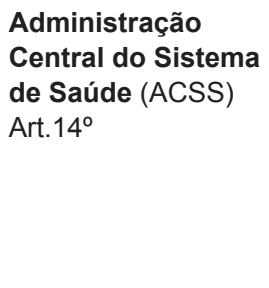 & $\begin{array}{l}\text { Assegurar a gestão dos recursos financeiros } \\
\text { e humanos do MS e do SNS, bem como das } \\
\text { instalações e equipamentos do SNS, proceder à } \\
\text { definição e implementação de políticas, normalização, } \\
\text { regulamentação e planeamento em saúde, nas áreas da } \\
\text { sua intervenção, em articulação com as administrações } \\
\text { regionais de saúde no domínio da contratação da } \\
\text { prestação de cuidados. }\end{array}$ \\
\hline
\end{tabular}
Atribuições relacionadas com Investigação

INFARMED Autoridade Nacional do Medicamento e Produtos de Saúde Art. $15^{\circ}$
Regular e supervisionar os sectores dos medicamentos de uso humano e dos produtos de saúde, segundo os mais elevados padrões de proteção da saúde pública e garantir o acesso dos profissionais da saúde e dos cidadãos a medicamentos e produtos de saúde de qualidade, eficazes e seguros
Assegurar a coordenação nacional da definição e desenvolvimento de programas de saúde, com base num sistema integrado de informação, articulando com os demais serviços e organismos do sistema de saúde, designadamente com o Instituto Nacional de Saúde Doutor Ricardo Jorge, I. P., em matéria de investigação científica e laboratorial.

Coordenar e centralizar a produção de informação e estatísticas dos prestadores de cuidados de saúde, nomeadamente produção, desempenho assistencial, recursos financeiros, humanos e outros.

Exercer, a nível nacional e internacional, no quadro do sistema comunitário de avaliação e supervisão de medicamentos e da rede de autoridades competentes da União Europeia, as funções de: (...)

iii) Laboratório de referência para a comprovação da qualidade de medicamentos no contexto da rede europeia de laboratórios oficiais de controlo (OMCL).

Promover e apoiar a investigação nos domínios da ciência e tecnologia das áreas da medicina transfusional, transplantação e medicina regenerativa, em articulação com o Instituto Nacional de Saúde Doutor Ricardo Jorge, I. P., e outras instituições nacionais e internacionais consideradas estratégicas para os objectivos propostos;

Desenvolver, promover e estimular a investigação e manter um sistema de informação sobre o fenómeno da droga e das toxicodependências.

\author{
Serviço de \\ Intervenção nos \\ Comportamentos \\ Aditivos e nas \\ Dependências \\ (SICAD) Art. $13^{\circ}$
}

Promover a redução do consumo de substâncias psicoactivas, a prevenção dos comportamentos aditivos e a diminuição das dependências. dádiva, colheita, análise, processamento, preservação, componentes sanguíneos, de órgãos, tecidos e células de origem humana. 
saúde, em especial nas áreas clínicas e de saúde pública. Há necessidade de promover investigação com uma abordagem adequada, com vista à implementação das políticas para os cuidados de saúde. A indústria, a academia e o SNS têm capacidades relevantes para a investigação e o desenvolvimento de novas terapias, e meios que podem beneficiar a saúde e os cuidados de saúde. Daí que os dirigentes do SNS, da comunidade científica e da indústria devessem colaborar para a criação de centros nacionais que, trabalhando em conjunto com os cidadãos, promovam o desenvolvimento de novas práticas, tecnologias e serviços. O relatório recomenda ainda o reforço do programa de investigação sobre saúde e das estratégias nacionais para a saúde pública (incluindo a promoção da saúde, a protecção da saúde e a prevenção da doença) para darem resposta aos grandes desafios da saúde, a nível nacional e da UE. Deverão ser assegurados quer o financiamento quer os recursos necessários para a investigação em saúde pública, incluindo a área dos serviços de saúde (um mínimo de $25 \%$ do financiamento para investigação sobre saúde deverá ser atribuído à saúde pública), tendo em conta a sua contribuição para o desenvolvimento económico do país. ${ }^{18}$

\section{Entidade Reguladora da Saúde}

Através de 'estudos e pareceres' e com recurso ao Sistema Nacional de Avaliação em Saúde (SINAS), a ERS tem adotado um papel de liderança no desenvolvimento da investigação avaliativa no setor da saúde, assumindo a investigação como um elemento essencial para conhecer o sector da saúde e os seus problemas, para orientar corretamente as atividades de supervisão comportamental dos regulados, desenhar intervenções regulatórias que visam moldar o comportamento dos agentes, promover a diminuição de assimetrias de informação e apoiar a decisão política. ${ }^{19}$

\section{Health Cluster Portugal}

O HCP foi criado em 2008 como uma organização privada sem fins lucrativos com o objetivo de "transformar Portugal num parceiro competitivo na investigação, conceção, desenvolvimento, fabrico e comercialização de produtos e serviços relacionados com a saúde, em mercados selecionados e em nichos tecnológicos, tendo como referência os mercados internacionais mais exigentes e relevantes, baseando-se no reconhecimento da sua qualidade, nível tecnológico e competência nas áreas de inovação". Este cluster é composto por mais de 120 entidades, incluindo hospitais, empresas e instituições científicas e tecnológicas. As suas atuais áreas de interesse estratégico incluem a saúde e o envelhecimento ativo, a medicina personalizada, o turismo de saúde e a e-Saúde. O HCP sugeriu à Comissão sobre "Um Futuro para a Saúde"18 que se reforce a investigação em colaboração com a indústria através de:

- Promoção da participação das universidades e dos hospitais, colaborando entre si e com empresas, em projetos de investigação e de desenvolvimento nacionais ou transnacionais, em áreas selecionadas de especializa- ção;

- Promoção de iniciativas destinadas a conseguir que as infraestruturas de investigação, a especialização e as tecnologias de universidades e hospitais sejam mais acessíveis a investigadores externos e a empresas, incentivando-se assim a transferência de conhecimentos/tecnologias, bem como a contratação de empresas de serviços de investigação e de desenvolvimento de ensaios clínicos;

- Melhoria dos níveis de qualificação e formação de recursos humanos / equipas a trabalhar em universidades e hospitais portugueses, no desenvolvimento de projetos de investigação translacional e clínica, bem como na gestão de inovação, propriedade intelectual, transferência de tecnologia e empreendedorismo.

\section{A INVESTIGAÇÃo EM SAÚde nOS PLANOS NACIO- NAIS DE SAÚDE}

Desde o fim do século $X X$ que a preparação e elaboração de PNS tem vindo a ser coordenada pela Direção Geral de Saúde e/ou Alto Comissariado da Saúde (organismo extinto em 2011).

Num primeiro PNS - Saúde um Compromisso: a estratégia de saúde para o virar do século (1998-2002) - a investigação tem direito a capítulo próprio. Estipula que "no que respeita a investigação em saúde torna-se necessário reavaliar todos os dispositivos e projetos de investigação em curso no âmbito do Ministério da Saúde, colaborar com os Ministérios da Educação e da Ciência e Tecnologia no seu desenvolvimento, incentivar a investigação em reforço da Estratégia de Saúde". Compromete o Ministério da Saúde com o desenvolvimento de uma política nacional de investigação em saúde. Identifica como prioritários estudos seroepidemiológicos para monitorizar o plano nacional de vacinação, investigação epidemiológica relativa à hepatite $\mathrm{C}$, investigação sobre os determinantes dos carcinomas do trato intestinal, estudos comportamentais relativos às dependências, estudos sobre determinantes das desigualdades em saúde no país, investigação sobre serviços de saúde. Aponta a necessidade de desenvolver um Observatório Nacional de Saúde no INSA e de integrar a formação em investigação clínica nos internatos das especialidades médicas. ${ }^{20}$

Em outubro de 2003, no âmbito dos trabalhos preparatórios do Plano Nacional de Saúde 2004 - 2010, foi realizada uma reunião temática sobre investigação em saúde, na sequência da qual se incluíram no PNS textos que por um lado enquadram a I\&D em saúde na história do SS português e por outro lançam importantes pistas de reflexão/ ação para um futuro próximo. ${ }^{5,6}$ A Fig. 2 sintetiza a 'análise da atualidade' feita na altura e a definição de intervenções e estratégias de futuro.

De salientar que, independentemente da decisão sobre a política de I\&D em ciências da saúde a seguir, se considerou, prioritária a realização de projetos sobre doenças crónicas e particularmente prevalentes e/ou incapacitantes, como as doenças cardiovasculares, o 


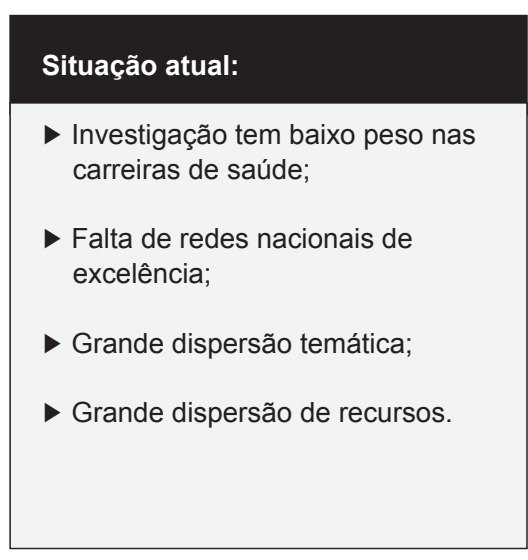

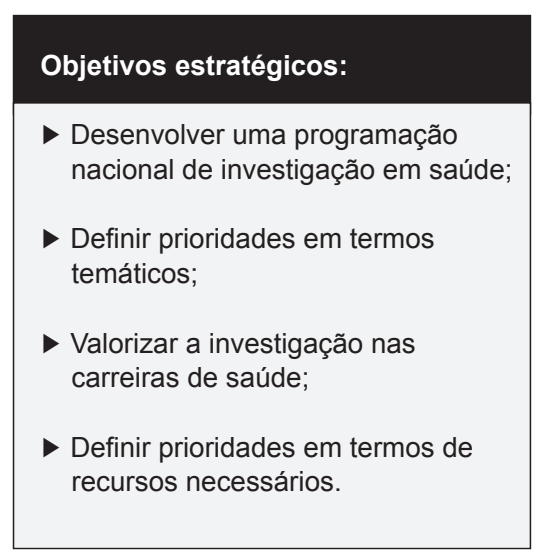

Figura 2 - Situação da I\&D em Portugal e orientações estratégicas. Adaptado de PNS 2004-2010.

cancro, a SIDA e outras doenças infecciosas, doenças mentais (incluindo comportamentos aditivos e problemas com eles relacionados) e sobre determinantes de saúde, como os estilos de vida ou as causas dos acidentes (de viação, trabalho e domésticos e de lazer). Foram ainda definidas outras áreas de investigação importantes nos contextos português e europeu atuais:

- Análise estratégica e prospectiva da evolução do sistema de saúde;

- Estudos da avaliação do desempenho dos serviços de saúde (acesso, qualidade, eficiência, resultados) em geral e dos seus recursos humanos em particular;

- Configuração e avaliação de sistemas de regulação e contratualização de saúde;

- Desenvolvimento da qualidade organizacional dos serviços de saúde;

- Análise da gestão da informação e do conhecimento em serviços de saúde;

- Estudo das percepções e da satisfação do cidadão em relação aos serviços de saúde, ao acesso à informação, e ao desempenho dos sistemas de reclamação;

- No referido documento ${ }^{6}$ ficou ainda definido, sob a responsabilidade do Departamento de Modernização e Recursos da Saúde, a operacionalização destas decisões;

- Contemplar, no percurso formativo dos profissionais de saúde, um período formal, obrigatório, de formação em Investigação Clínica;

- Na avaliação dos serviços de saúde deverão valorizar as atividades de investigação desses mesmos serviços. Na preparação do PNS 2011-2016, para além do parecer solicitado à FCT e referido na Fig. 1, foi realizada uma análise especializada sobre Investigação Científica e Plano Nacional de Saúde (Roteiro de Intervenção - Os Roteiros de Intervenção são estudos de autor solicitados a peritos nacionais com o objetivo de contribuir com a meIhor evidência e conhecimentos para o Plano Nacional de Saúde (PNS) 2012-2016. Estes roteiros são documentos, de autor, que fornecem um modelo concetual, um ponto de situação e recomendações para cada um dos temas. As informações expressas nos mesmos traduzem os conhecimentos e opiniões dos seus autores, não tendo sido alvo de qualquer alteração pela Direção-Geral da Saúde. (http:// pns.dgs.pt/roteiros-de-intervencao-do-plano-nacional-de-saude/)), a qual ${ }^{21}$ pouco influenciou o texto de um plano com referências superficiais à investigação.

As principais recomendações do PNS 2012-2016 quanto à investigação são as especificadas no âmbito dos programas nacionais prioritários (Programa Nacional para a Prevenção e Controlo do Tabagismo; Programa Nacional para a Promoção da Alimentação Saudável; Programa Nacional para as Doenças Cérebro-cardiovasculares; Programa Nacional para as Doenças Oncológicas; Programa Nacional para a Diabetes; Programa Nacional para a Saúde Mental; Programa Nacional para as Doenças Respiratórias; Programa Nacional para a Infeção VIH/SIDA; Programa Nacional de Prevenção e Controlo de Infeção e Resistência aos Antimicrobianos).

\section{CONCLUSÕES E RECOMENDAÇÕES}

As orientações estratégias do PNS 2004 - 2010 são as que mais se aproximam de uma política de investigação para a saúde sem que, no entanto, as questões então abordadas tenham sido desenvolvidas de uma forma abrangente ou a posteriori implementadas pelas instituições que têm responsabilidades sobre a investigação científica no setor da saúde.

A investigação para a saúde não tem sido uma prioridade e o impacto desta insuficiência pode tomar proporções consideráveis, afetando os processos de tomada de decisão, a identificação precisa dos grupos de risco e grupos-alvo para determinadas intervenções, a abordagem às doenças e, por exemplo, a efetividade associada a determinada intervenção. ${ }^{5,6}$ Esta situação é também limitadora do potencial desenvolvimento do complexo económico associado à saúde. ${ }^{22}$

Em Portugal, muito poucas reformas importantes foram precedidas de documentos de análise, evidenciando o conhecimento existente que fundamenta as decisões, estabelecendo resultados esperados, de forma objetiva e quantificada, estabelecendo a forma a adoptar para avaliar o impacto dessa reforma ou ainda beneficiaram de debate técnico e publico alargado. A ausência destas linhas de 
referência compromete a monitorização e avaliação prospectiva e enfraquece a implementação. ${ }^{23}$

Importa salientar que a reflexão sobre esta matéria tem sido crescente. Ilustram-no por exemplo os já citados documentos Diagnóstico do Sistema de Investigação e Inovação: desafios, forças e fraquezas rumo e 2020 e Estratégia Nacional de Investigação e Inovação para uma Especialização Inteligente EI\&I 2014-2020. ${ }^{15,24}$ Concretamente no domínio da saúde, a criação do Fundo para a Investigação em Saúde que atribuiu recentemente (novembro de 2015) os primeiros fundos destinados a investigação, como resultado de uma cooperação profícua entre o MS e o Ministério da Educação e Ciência (através da FCT), apresenta-se como um dos instrumentos para estimular a investigação para a saúde em Portugal. ${ }^{25}$ Por outro lado, a determinação de que os hospitais do SNS tenham um serviço de Serviço de Investigação, Epidemiologia Clínica e de Saúde Pública Hospitalar ${ }^{26}$ demonstra a consciência da necessidade de haver uma grande intervenção do MS na promoção da investigação para a saúde em Portugal. ${ }^{25}$

Em Portugal, tem vindo a crescer a tomada de consciência de que a solução para os problemas da sustentabilidade e relevância dos sistemas de saúde requer uma melhor compreensão da relação complexa entre determinantes da saúde, novas tecnologias em saúde e a economia, compreensão essa que depende de um investimento racional na investigação para a saúde. ${ }^{27}$ Essa investigação deverá estar enquadrada num SNIS, o qual deve estar integrado na política científica do país, em particular na política de investigação para a saúde, e cuja finalidade principal é produzir novos conhecimentos de elevada qualidade que possam ser usados para promover, restaurar e/ou manter o estado de saúde de indivíduos ou populações, incluindo os mecanismos para encorajar a utilização da investigação. ${ }^{28}$

Contudo, a realidade demonstra a não existência de um plano de investigação científica para a saúde em Portugal, o qual possa pôr em franca articulação os diferentes atores que intervêm nesta matéria. A ausência de uma análise aprofundada do que se tem feito em investigação científica para a saúde, de uma avaliação do que se tem implementado ao longo dos tempos, dificulta a identificação dos aspetos chave que podem trazer contributos fundamentais ao processo de produção de uma política de investigação para a saúde.

Na convicção de que adoptar uma estratégia de incentivo à investigação e desenvolvimento em saúde é, por excelência, uma mais-valia para o sistema de saúde português que poderá ter reflexos importantes no capital de saúde da população, 5,6 com base nas considerações tecidas nas páginas anteriores, e considerando as recomendações a níveis global e europeu, os autores propõe cinco sugestões estratégicas em matérias de investigação para a saúde em Portugal:

1. Avaliação dos planos de investigação para a saúde e aplicação das evidências científicas nos processos de decisão do Governo e instituições envolvidas na execução da política nacional de saúde;

2. Definição de uma política nacional de investigação para a saúde com princípios orientadores e prioridades decorrentes do contexto do país;

3. Aplicação de uma abordagem transversal e abrangente que cubra não somente os aspectos biomédicos e dos sistemas de saúde, mas também as determinantes ambientais, económicas e sociais da saúde;

4. Estabelecimento de planos institucionais e programas de investigação interdisciplinares que tenham em conta a pluralidade metodológica e que criem oportunidades para iniciativas de investigadores de disciplinas afins;

5. Desenvolvimento de uma classificação consensual de investigação para saúde em Portugal, inspirada nas recomendações a nível global e europeu.

\section{AGRADECIMENTOS}

AAna Cristina Garcia e Cláudio M. Soares pela colaboração na redação do artigo. A Rosa Ferrinho pelo apoio à pesquisa bibliográfica. A Ana Rocha pela elaboração das figuras.

\section{CONFLITOS DE INTERESSE}

Os autores declaram não terem qualquer conflito de interesse relativamente ao presente artigo.

\section{FONTES DE FINANCIAMENTO}

Os autores declaram não ter recebido subsídios ou bolsas para a elaboração do artigo.

\section{REFERÊNCIAS}

1. Guerreiro CS, Hartz Z, Sambo L, Conceição MC, Dussault G, Russo G, et al. Política de Investigação Científica em Portugal: I - Enquadramento Europeu e Nacional. Acta Med Port. 2017;30:141-7.

2. Heitor M. Ciência e conhecimento na modernização de Portugal: a formulação de políticas públicas na superação do atraso científico e na democratização do acesso ao conhecimento. Capítulo do livro"40 Anos de Políticas de Ciência e Ensino Superior em Portugal”. Lisboa: Ed. Almedina; 2015. [consultado 2016 jun 16]. Disponível em http://www. manifesto2015.com/docs/capitulo-politicas-C-T-MHeitor-11mai2015. pdf.

3. Research and Innovation Observatory - Horizon 2020 Policy Support Facility. [consultado 2015 out 16]. Disponível em http://erawatch.jrc. ec.europa.eu/erawatch/opencms/information/country_pages/pt/country ?section=ResearchPolicy\&subsection=RecentResearchPolicyDev.
4. Portugal - RIO Country Report. [consultado 2016 jul 04]. Disponível em https://rio.jrc.ec.europa.eu/en/country-analysis/Portugal/country-report.

5. Portugal, Ministério da Saúde. Plano Nacional de Saúde 2004-2010 Prioridades. Lisboa: Ministério da Saúde; 2004.

6. Portugal, Ministério da Saúde. Plano Nacional de Saúde 2004-2010 Orientações Estratégicas. Lisboa: Ministério da Saúde; 2004.

7. Direção-Geral de Estatísticas da Educação e da Ciência. Estatísticas de investigação e desenvolvimento. 20 principais indicadores de despesa e recursos humanos em I\&D. Inquérito ao potencial científico e tecnológico nacional. 2013. [consultado 2016 jun 16]. Disponível em http://www.dgeec.mec.pt/np4/571.html.

8. Direção-Geral de Estatísticas da Educação e da Ciência. Despesa em I\&D: Portugal e EU-15. 2014. [consultado 2016 jun 16]. Disponível em http://www.dgeec.mec.pt/np4/460.html. 
9. Villax P, Fonseca C, Outeiro T, Lucena D, Arantes e Oliveira N. Capítulo 1 - Financiamento da Ciência em Portugal pp 27-37 em Assembleia da República, Comissão de Educação e Ciência (2010). A ciência em Portugal - Relatório. Lisboa: Assembleia da República; 2010.

10. Fundação para a Ciência e Tecnologia. Portuguese roadmap for research infrastructures 2014-2020. Lisboa: FCT; 2014.

11. Portugal, Ministério da Ciência, Tecnologia e Ensino Superior. A Participação da Fundação para a Ciência e a Tecnologia-MCTES na Promoção da Investigação em Saúde. Lisboa, Ministério da Ciência, Tecnologia e Ensino Superior, 2010. [consultado 2016 jun 16]. Disponivel em http://pns.dgs.pt/files/2010/10/mctes.pdf.

12. Carvalho-Oliveira I, Calheiros JM, Pereira Miguel J. A investigação em saúde e o ministério da saúde. In Campos AC e Simões J, Coord. 40 anos de abril na saúde. Lisboa: Almedina; 2015. Capítulo 7, p. 233-53,

13. Pereira Miguel, J. A saúde em tempos de transição (1968-1974). Intervenção proferida a 23.10-2008 no ciclo de debates promovido pelo Centro Nacional de Cultura, Grémio literário e Círculo Eça de Queirós sobre o tema "Tempos de Transição (1968-1974)". [consultado a 2016 jun 16]. Disponível em http://www.academianacionalmedicina. pt/Backoffice/UserFiles/File/Documentos/Sa\%C3\%BAde\%20na\%20 Transi\%C3\%A7\%C3\%A3o.pdf.

14. Instituto Nacional de Saúde Doutor Ricardo Jorge. Aliando excelência científica com relevância na promoção da saúde e controlo da doença: para uma Agenda Portuguesa de I\&D em Saúde. Lisboa: INSA, 2011.

15. Fundação para Ciência e Tecnologia. Diagnóstico do sistema de investigação e inovação: desafios, forças e fraquezas rumo e 2020. Lisboa: FCT; 2013.

16. IAPMEI, FCT, adi, COMPETE, QREN, EU. Diagnóstico de Apoio às Jornadas de Reflexão Estratégica; Documento de trabalho n², Eixo temático 5 - Saúde, Bem-estar e Território; Saúde. Estratégia Nacional de Investigação e Inovação para uma Especialização Inteligente 20142020

17. Campos AC, Simões J. O percurso da saúde: Portugal na Europa. Capítulo 3 - A investigação científica sobre saúde na União Europeia e em Portugal. Lisboa: Almedina; 2011. p. 95-110.
18. Crisp N, Coord. Um Futuro para a Saúde. Todos temos um papel a desempenhar. Lisboa: Fundação Calouste Gulbenkian; 2014.

19. Simões J, Carneiro C. Investigação avaliativa na regulação da saúde. An Inst Higiene Med Trop. 2014;13:81-6.

20. Ministério da Saúde. Saúde, um compromisso. A estratégia de saúde para o virar do século 1998-2002. Lisboa: Ministério da Saúde; 1999.

21. Lobo Antunes J. Investigação científica e plano nacional de saúde. Lisboa: Alto Comissariado da Saúde; 2010.

22. Ferrinho P, de Sousa B, Tavares A, Nogueira P, Portugal R. Sistema de saúde na região de saúde de Lisboa e Vale do Tejo: para além dos centros de saúde e hospitais. Acta Med Port. 2012;25:375-88.

23. Amendoeira J. Políticas de saúde em Portugal e desigualdades. Departamento de Sociologia - Seminários Temáticos Políticas Públicas e Desigualdades. Escola de Enfermagem, Instituto Politécnico de Santarém e CesNova, FCSH, UNL, 2009. [consultado 2016 jun 16]. Disponível em http://repositorio.ipsantarem.pt/bitstream/10400.15/86/1 Pol\%C3\%ADticas\%20de\%20sa\%C3\%BAde\%20em\%20Portugal\%20 e\%20desigualdades.pdf.

24. IAPMEI, FCT, ANI, COMPETE. Estratégia Nacional de Investigação e Inovação para uma Especialização Inteligente EI\&I 2014-2020. Versão de Novembro de 2014.

25. Leal da Costa F. Fundo para a Investigação em Saúde. Intervenção proferida a 17.11.2015 na apresentação dos projetos selecionados ao Fundo para a Investigação em Saúde. [consultado 2016 jun 16]. Disponível em http://www.portaldasaude.pt/portal/ conteudos/a+saude+em+portugal/ministerio/comunicacao/ discursos+e+intervencoes/fistselecao.htm.

26. Diário da República, 2. ${ }^{\text {a }}$ série. N. ${ }^{\circ} 126$ - 1 de Julho de 2015. Despacho (extrato) n. ${ }^{\circ}$ 7216/2015. Lisboa: Assembleia da República; 2015.

27. Pardes $\mathrm{H}$, Manton KG, Lander ES, Tolley HD, Ullian AD, Palmer $\mathrm{H}$. Effects of medical research on health care and the economy. Science. 1999;283:36-7.

28. Pang T, Sadana R, Hanney S, Bhutta ZA, Hyder AA, Simon J. Knowledge for better health - a conceptual framework and foundation for health research systems. Bull WHO. 2003;81:815-20. 